\title{
Transmission Quality Evaluation for a Class of Four-limb Parallel Schönflies-motion Generators with Articulated Platforms
}

\author{
Guanglei $\mathrm{Wu}^{1}$ and Shaoping Bai ${ }^{2}$ and Stéphane $\mathrm{Caro}^{3}$ \\ ${ }^{1}$ School of Mechanical Engineering, Dalian University of Technology, Dalian \\ 116024, China, e-mail: gwu@dlut.edu.cn \\ ${ }^{2}$ Department of Mechanical and Manufacturing Engineering, Aalborg University, \\ 9220 Aalborg, Denmark,e-mail: shb@make.aau.dk \\ ${ }^{3}$ CNRS, Laboratoire des Sciences du Numérique de Nantes, UMR CNRS 6004, \\ Ecole Centrale de Nantes, 44321 Nantes, France, e-mail: stephane.caro@ls2n.fr
}

\begin{abstract}
This paper investigated the motion/force transmission quality for a class of parallel Schönflies-motion generators built with four identical RR $\Pi$ RR-type limbs. It turns out that the determinant of the forward Jacobian matrices for this class of parallel robots can be expressed as the scalar product of two vectors, the first vector being the cross product of the four unit vectors along the parallelograms, the second one being related to the rotation of the mobile platform. The pressure angles, derived from the determinants of forward and inverse Jacobians, respectively, are used for the evaluation of the transmission quality of the robots. Four robots are compared based on the proposed method as illustrative examples.
\end{abstract}

Key words: Schönflies motion, Jacobian, pressure angle, transmission.

\section{Introduction}

Parallel robots performing Schönflies motions are well adapted to high-speed pickand-place (PnP) operations [10, 4], thanks to their lightweight architecture and high stiffness. A typical robot is the Quattro robot [1] by Adept Technologies Inc., the fastest industrial robot available. Its latest version can reach an acceleration up to $15 G$ with a $2 \mathrm{~kg}$ payload, allowing to accomplish four standard PnP cycles per second. Its similar version is the $\mathrm{H} 4$ robot [9] that consists of four identical limbs and an articulated traveling plate [6]. Recently, the Veloce. robot [2] with a different articulated platform that is connected by a screw pair has been developed. Besides, the four-limb robots with single-platform architecture have also been reported [13, 14]. Four-limb parallel robots with an articulated mobile platform are displayed in Fig. 1. It is noteworthy that the $\mathrm{H} 4$ robot with the modified mobile platform can be mounted vertically instead of the horizontal installation for the reduced mounting space, to provide a rotation around an axis of vertical direction, which is named as "V4" for convenience in the following study.

In the design and analysis of a manipulator, its kinematic Jacobian matrix plays an important role, since the dexterity/manipulability of the robot can be evaluated by the condition number of Jacobians as well as the accuracy/torque capability [8] be- 
tween the actuators and end-effector. On the other hand, a problem usually encountered in this procedure is that the parallel manipulators with mixed input or/and output motions, i.e., compound linear and angular motions, will result in dimensionally inhomogeneous Jacobians, thus, the conventional performance indices associated with the Jacobian matrix, such as norm or condition number, will lack in physical significance [7]. As far as Schönflies-motion generators are concerned, their endeffector generates a mixed motion of three translations and one rotation (3T1R), for which the terms of the kinematic Jacobian matrix do not have the same units. A common approach to overcome this problem is to introduce a characteristic length [3] to homogenize the Jacobian matrix, whereas, the measurement significantly depends on the choice of the characteristic length that is not unique, resulting in biased evaluation, although a "best" one can be found by optimization technique [5]. Alternatively, an efficient approach to accommodate this dimensional inhomogeneity is to adopt the concept of the virtual coefficient, namely, the transmission index, which is closely related to the transmission/pressure angle. The pressure angle based transmission index will be adopted in this work.

This paper presents a uniform evaluation approach for transmission quality of a family of four-limb 3T1R parallel robots with articulated mobile platforms. The pressure angles, derived from the forward and inverse Jacobians straightforward, are used for the evaluation of the transmission quality of the robots. The defined transmission index is illustrated with four robot counterparts for the performance evaluation and comparison.

\section{Manipulator Architecture}

Figure 2(a) depicts a simplified CAD model of the parallel Schönflies-motion generator, which is composed of four identical RR $R R^{1}$-type limbs connecting the base and an articulated mobile platform (MP). The generalized base platform and the different mobile platforms of the four robots are displayed in Figs. 2(b) and 2(c), respectively.

The global coordinate frame $\mathscr{F}_{b}$ is built with the origin located at the geometric center of the base platform. The $x$-axis is parallel to the segment $A_{2} A_{1}\left(A_{3} A_{4}\right)$, and the $z$-axis is normal to the base-platform plane pointing upwards. The moving coordinate frame $\mathscr{F}_{p}$ is attached to the mobile platform and the origin is at the geometric center, where $X$-axis is parallel to segment $C_{2} C_{1}\left(C_{3} C_{4}\right)$. Vectors $\mathbf{i}, \mathbf{j}$ and $\mathbf{k}$ represent the unit vectors of $x$-, $y$ - and $z$-axis, respectively. The axis of rotation of the $i$ th actuated joint is parallel to unit vector $\mathbf{u}_{i}=\mathbf{R}_{z}\left(\alpha_{i}\right) \mathbf{i}$, where $\mathbf{R}$ stands for the rotation matrix, and $\alpha_{1}=-\alpha_{2}=\alpha-\pi / 2, \alpha_{3}=-\alpha_{4}=\beta+\pi / 2$. Moreover, unit vectors $\mathbf{v}_{i}$ and $\mathbf{w}_{i}$ are parallel to the segments $\overline{A_{i} B_{i}}$ and $\overline{B_{i} C_{i}}$, respectively, namely, the unit vectors along the proximal and distal links, respectively.

\footnotetext{
${ }^{1} \mathrm{R}$ and $\Pi$ stand for revolute joint and parallelogram ( $\Pi$ joint), respectively, and the underlined letter indicates the actuated joint.
} 


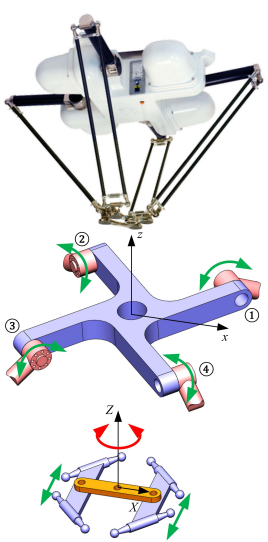

(a)

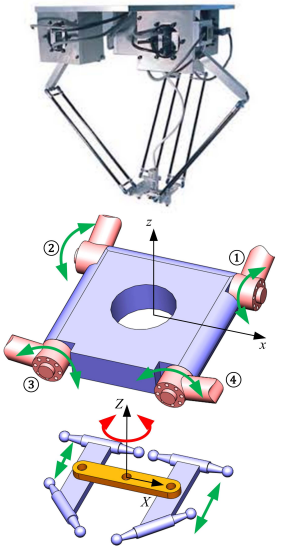

(b)

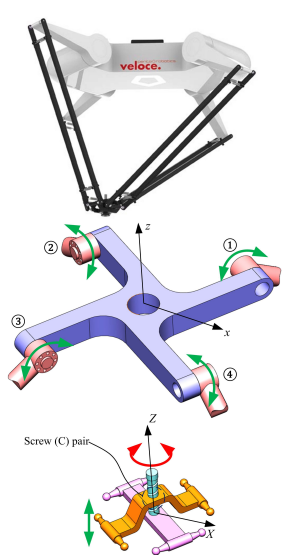

(c)

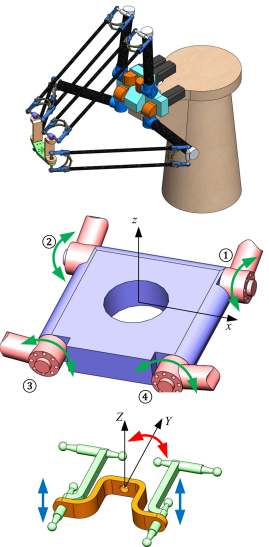

(d)

Fig. 1 The four-limb PnP robots with different base and mobile platforms: (a) Quattro [1]; (b) H4 [9]; (c) Veloce. [2]; (d) "V4" [12].

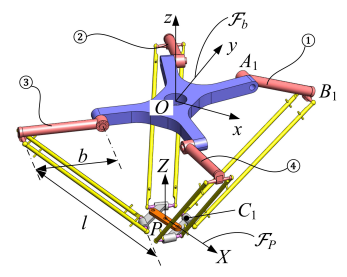

(a)

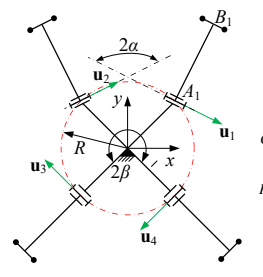

(b)

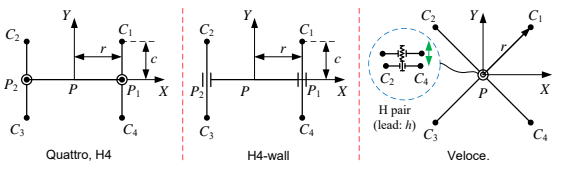

(c)

Fig. 2 The parameterization of the four-limb robots: (a) simplified CAD model; (b) a generalized base platform; (c) three different mobile platforms for the four robots.

\section{Kinematics and Jacobian Matrix of the Robots}

The Cartesian coordinates of points $A_{i}$ and $B_{i}$ expressed in the frame $\mathscr{F}_{b}$ are respectively derived by

$$
\begin{aligned}
\mathbf{a}_{i} & =R\left[\cos \eta_{i} \sin \eta_{i} 0\right]^{T} \\
\mathbf{b}_{i} & =b \mathbf{v}_{i}+\mathbf{a}_{i} ; \mathbf{v}_{i}=\mathbf{R}_{z}\left(\alpha_{i}\right) \mathbf{R}_{x}\left(\theta_{i}\right) \mathbf{j}
\end{aligned}
$$

where $\eta_{i}=(2 i-1) \pi / 4, i=1, \ldots, 4$, and $\theta_{i}$ is the input angle.

Let the mobile platform pose be denoted by $\boldsymbol{\chi}=\left[\begin{array}{l}\mathbf{p}^{T} \phi\end{array}\right]^{T}, \mathbf{p}=\left[\begin{array}{ll}x y & y\end{array}\right]^{T}$, the Cartesian coordinates of point $C_{i}$ in frame $\mathscr{F}_{b}$ are expressed as

$$
\mathbf{c}_{i}=\left\{\begin{array}{l}
\operatorname{sgn}\left(\cos \eta_{i}\right) r \mathbf{R}_{z}(\phi) \mathbf{i}+\operatorname{sgn}\left(\sin \eta_{i}\right) c \mathbf{j}+\mathbf{p}, \quad \text { Quattro }(\mathrm{H} 4) \\
-\operatorname{sgn}\left(\cos \eta_{i}\right) r \mathbf{R}_{y}(\phi) \mathbf{i}+\operatorname{sgn}\left(\cos \eta_{i}\right) c \mathbf{j}+\mathbf{p}, \quad \text { V4 } \\
r \mathbf{R}_{z}\left(\eta_{i}\right) \mathbf{i}+\bmod (i, 2) h \phi /(2 \pi) \mathbf{k}+\mathbf{p}, \quad \text { Veloce }
\end{array}\right.
$$


where $\operatorname{sgn}(\cdot)$ stands for the sign function of $(\cdot)$, and mod stands for the modulo operation, $h$ being the lead of the screw pair of the Veloce. robot.

The inverse geometric problem has been well documented [10]. It can be solved from the following the kinematic constraint equations:

$$
\left(\mathbf{c}_{i}-\mathbf{b}_{i}\right)^{T}\left(\mathbf{c}_{i}-\mathbf{b}_{i}\right)=l^{2}, \quad i=1, \ldots, 4
$$

Differentiating Eq. (4) with respect to time, one obtains

$$
\dot{\phi} r \mathbf{w}_{i}^{T} \mathbf{s}_{i}+\mathbf{w}_{i}^{T} \dot{\mathbf{p}}=\dot{\theta}_{i} b \mathbf{w}_{i}^{T}\left(\mathbf{u}_{i} \times \mathbf{v}_{i}\right)
$$

with

$$
\mathbf{w}_{i}=\frac{\mathbf{c}_{i}-\mathbf{b}_{i}}{l} ; \quad \mathbf{s}_{i}= \begin{cases}\operatorname{sgn}\left(\cos \eta_{i}\right) \mathbf{R}_{z}(\phi) \mathbf{j}, & \text { Quattro }(\mathrm{H} 4) \\ \operatorname{sgn}\left(\cos \eta_{i}\right) \mathbf{R}_{y}(\phi) \mathbf{k}, & \text { V4 } \\ \bmod (i, 2) h \phi /(2 \pi) \mathbf{k}, & \text { Veloce }\end{cases}
$$

Equation (5) can be cast in a matrix form, namely,

$$
\mathbf{A} \dot{\boldsymbol{\chi}}=\mathbf{B} \dot{\boldsymbol{\theta}}
$$

with

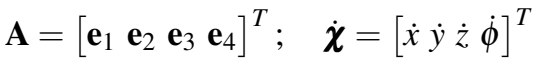

$$
\begin{aligned}
& \mathbf{B}=\operatorname{diag}\left[\begin{array}{llll}
h_{1} & h_{2} & h_{3} & h_{4}
\end{array}\right] ; \quad \dot{\boldsymbol{\theta}}=\left[\begin{array}{llll}
\dot{\theta}_{1} & \dot{\theta}_{2} & \dot{\theta}_{3} & \dot{\theta}_{4}
\end{array}\right]^{T}
\end{aligned}
$$

where $\mathbf{A}$ and $\mathbf{B}$ are the forward and inverse Jacobian matrices, respectively, and

$$
\mathbf{e}_{i}=\left[\begin{array}{lll}
\mathbf{w}_{i}^{T} & r \mathbf{w}_{i}^{T} \mathbf{s}_{i}
\end{array}\right]^{T} ; \quad h_{i}=b \mathbf{w}_{i}^{T}\left(\mathbf{u}_{i} \times \mathbf{v}_{i}\right)
$$

As along as $\mathbf{A}$ is nonsingular, the kinematic Jacobian matrix is obtained as

$$
\mathbf{J}=\mathbf{A}^{-1} \mathbf{B}
$$

According to the inverse Jacobian matrix, each limb can have two working modes, which is characterized by the sign " $-/+$ " of $h_{i}$. In order for the robot not to reach any serial singularity, the mode $h_{i}<0, i=1, \ldots, 4$, is selected as the working mode for all the robots.

\section{Transmission Quality Analysis}

Our interests are the transmission quality, which is related to the robot Jacobian. The determinant $|\mathbf{B}|$ of the inverse Jacobian matrix $\mathbf{B}$ is expressed as

$$
|\mathbf{B}|=\prod_{i=1}^{4} h_{i}=b^{4} \prod_{i=1}^{4} \mathbf{w}_{i}^{T}\left(\mathbf{u}_{i} \times \mathbf{v}_{i}\right)
$$


sequentially, the pressure angle $\mu_{i}$ associated with the motion transmission in the $i$ th limb, i.e., the motion transmitted from the actuated link to the parallelogram, is defined as:

$$
\mu_{i}=\cos ^{-1} \mathbf{w}_{i}^{T}\left(\mathbf{u}_{i} \times \mathbf{v}_{i}\right), \quad i=1, \ldots, 4
$$

namely, the pressure angle between the velocity of point $B_{i}$ along the vector of $\mathbf{u}_{i} \times$ $\mathbf{v}_{i}$ and the pure force applied to the parallelogram along $\mathbf{w}_{i}$, as shown in Fig. 3(a).

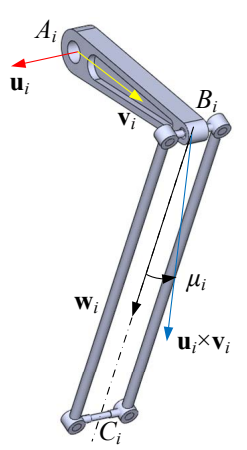

(a)

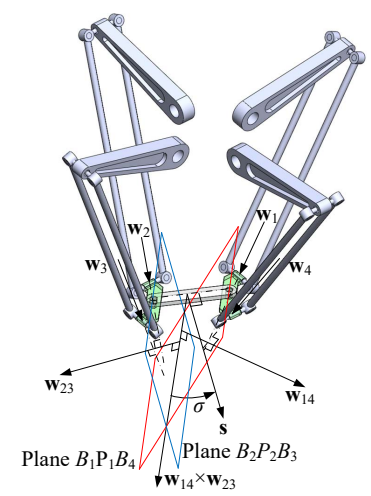

(b)

Fig. 3 The pressure angles of the four-limb robots in the motion/force transmission: (a) $\mu_{i}$ for all robots; (b) $\sigma$ for Quattro.

Likewise, with the Laplace expansion, the determinant $|\mathbf{A}|$ of the forward Jacobian matrix $\mathbf{A}$ is simplified as

$$
|\mathbf{A}|=\left\{\begin{array}{l}
-2 r\left(\mathbf{w}_{14} \times \mathbf{w}_{23}\right)^{T} \mathbf{s}, \mathbf{s}=\mathbf{R}_{z}(\phi) \mathbf{j} ; \quad \text { Quattro }(\mathrm{H} 4) \\
2 r\left(\mathbf{w}_{14} \times \mathbf{w}_{23}\right)^{T} \mathbf{s}, \mathbf{s}=\mathbf{R}_{y}(\phi) \mathbf{k} ; \quad \text { V4 } \\
h \phi /(2 \pi)\left(\mathbf{w}_{13} \times \mathbf{w}_{24}\right)^{T} \mathbf{k} ; \quad \text { Veloce. }
\end{array}\right.
$$

where $\mathbf{w}_{m n}=\mathbf{w}_{m} \times \mathbf{w}_{n}$. Taking the Quattro robot as an example, the pressure angle $\sigma$ amongst limbs, namely, the force transmitted from the end-effector to the passive parallelograms in the other limbs, provided that the actuated joints in these limbs are locked, is derived below:

$$
\sigma=\cos ^{-1} \frac{\left(\mathbf{w}_{14} \times \mathbf{w}_{23}\right)^{T} \mathbf{s}}{\left\|\mathbf{w}_{14} \times \mathbf{w}_{23}\right\|}
$$

wherefrom the geometrical meaning of angle $\sigma$ can be interpreted as the angle between the minus $Y$-axis (s is normal to segment $\overline{P_{1} P_{2}}$ ) and the intersection line of planes $B_{1} P_{1} B_{4}$ and $B_{2} P_{2} B_{3}$, where plane $B_{1} P_{1} B_{4}\left(B_{2} P_{2} B_{3}\right)$ is normal to the common perpendicular line between the two skew lines along $\mathbf{w}_{1}$ and $\mathbf{w}_{4}\left(\mathbf{w}_{2}\right.$ and $\left.\mathbf{w}_{3}\right)$, as depicted in Fig. 3(b). To illustrate the angle $\sigma$ physically, $\left(\mathbf{w}_{14} \times \mathbf{w}_{23}\right)^{T} \mathbf{s}$ can be rewritten in the following form: 


$$
\begin{aligned}
\left(\mathbf{w}_{14} \times \mathbf{w}_{23}\right)^{T} \mathbf{s} & =\mathbf{w}_{14}^{T}\left[\mathbf{w}_{3}\left(\mathbf{w}_{2} \cdot \mathbf{s}\right)-\mathbf{w}_{2}\left(\mathbf{w}_{3} \cdot \mathbf{s}\right)\right] \\
& =\mathbf{w}_{23}^{T}\left[\mathbf{w}_{4}\left(\mathbf{w}_{1} \cdot \mathbf{s}\right)-\mathbf{w}_{1}\left(\mathbf{w}_{4} \cdot \mathbf{s}\right)\right]
\end{aligned}
$$

The angle $\sigma$ now can be interpreted as the pressure angle between the velocity in the direction of $\mathbf{w}_{1} \times \mathbf{w}_{4}$ and the forces along $\mathbf{w}_{2} \times \mathbf{w}_{3}$ imposed by the parallelograms in limbs 2 and 3 to point $P$, under the assumption that the actuated joints in limbs 1 and 4 are locked simultaneously. The same explanation is applicable for the case when the actuated joints in limbs 2 and 3 are locked.

By the same token, the pressure angle for the remaining robot counterparts can be defined. Consequently, the motion $\kappa$ and force $\zeta$ transmission indices (TI) in a prescribed configuration are defined as the minimum value of the cosine of the pressure angles, respectively,

$$
\kappa=\min \left(\left|\cos \mu_{i}\right|\right), i=1, \ldots, 4 ; \quad \zeta=|\cos \sigma|
$$

To this end, the local transmission index (LTI) [11] is defined as

$$
\eta=\min \{\kappa, \zeta\}=\min \left\{\left|\cos \mu_{i}\right|,|\cos \sigma|\right\} \in[0,1]
$$

The larger the value of the index $\eta$, the better the transmission quality of the manipulator. This index can also be applicable for singularity measurement, where $\eta=0$ means singular configuration.

\section{Transmission Evaluation of PnP Robots}

In this section, the transmission index over the regular workspace, for the Quattro, $\mathrm{H} 4$, Veloce. and V4 robots, will be mapped to analyzed their motion/force transmission qualities. According to the technical parameters of the Quattro robot [1], the parameters of the robots' base and mobile platforms are given in Table 1, and other parameters are set to $R=275 \mathrm{~mm}, b=375 \mathrm{~mm}$ and $l=800 \mathrm{~mm}$, respectively.

Table 1 Geometrical parameters of the base and mobile platforms of the four-limb robots.

\begin{tabular}{ll}
\hline robots $\quad$ base & mobile platform \\
\hline Quattro $\alpha=-\pi / 4, \beta=3 \pi / 4$ & $r=80 \mathrm{~mm}, c=70 \mathrm{~mm}$ \\
H4, V4 $\alpha=0, \beta=\pi / 2$ & $r=80 \mathrm{~mm}, c=70 \mathrm{~mm}$ \\
Veloce. $\alpha=-\pi / 4, \beta=3 \pi / 4$ & $r=100 \mathrm{~mm}, \gamma=(2 i-1) \pi / 4, h$ \\
\hline
\end{tabular}

The LTI isocontours of the four robots with different rotation angles of mobile platform are visualized in Fig. 4, from which it is seen that the minimum LTI of the Quattro and Veloce. robots are much higher than those of H4 and V4. Moreover, the volumes of the formers with LTI $\geq 0.7$ are larger, to formulate larger operational workspace with high transmission quality. This means that the four-limb robots with a fully symmetrical structure have much better transmission performance than the 
asymmetric robot counterparts. Another observation is that the transmission performance of the robots decreases with the increasing MP rotation angle.

As displayed in Fig. 4(a), the transmission index of the Quattro robot have larger values in the central region, which admits a singularity-free workspace with rotational capability $\phi= \pm 45^{\circ}$. Similarly, Fig. 4(c) shows that the Veloce. robot can also have a high-transmission workspace free of singularity with smaller lead of screw pair, which means that this type of mobile platform allows the robot to have high performance in terms of transmission quality and rotational capability of fullcircle rotation. By contrast, the asymmetric $\mathrm{H} 4$ and V4 robots result in relatively small operational workspace and relatively low transmission performance, as illustrated in Figs. 4(b) and 4(d), but similar mechanism footprint ratio with same link dimensions and close platform shapes.
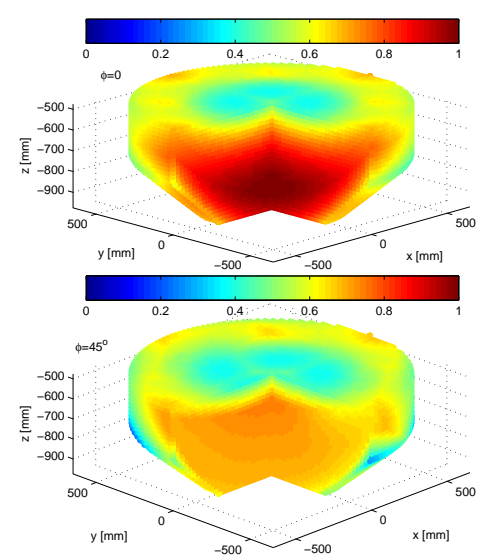

(a)

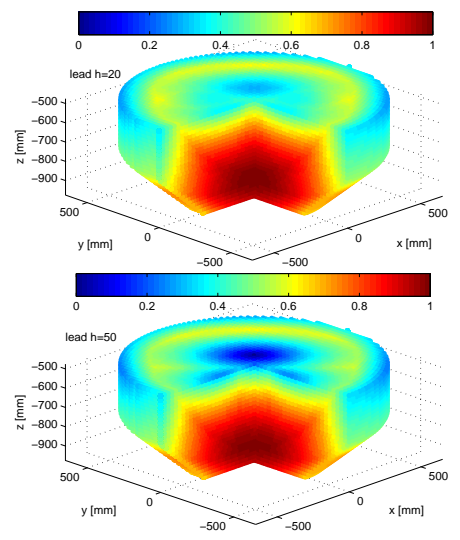

(c)

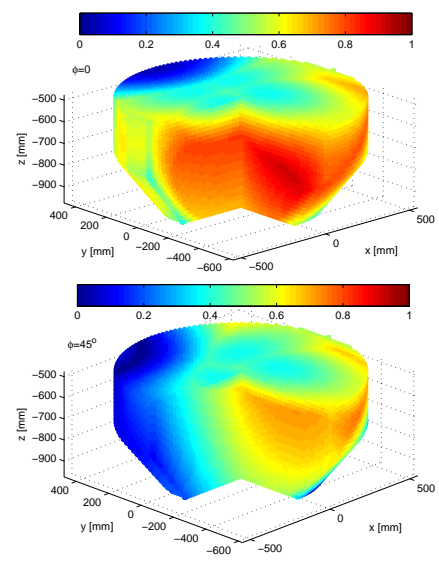

(b)

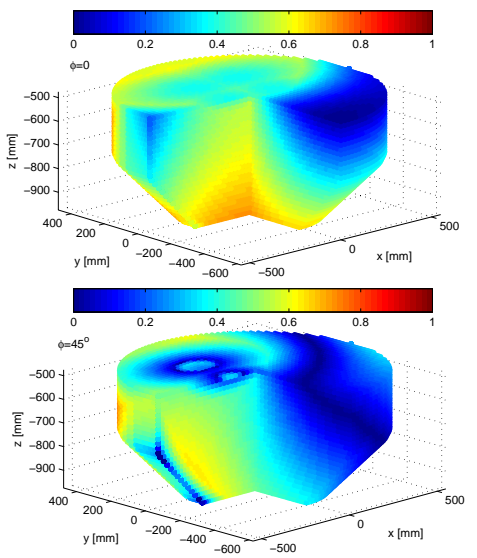

(d)

Fig. 4 The LTI isocontours of the robots: (a) Quattro, $\phi=0$ and $\phi=45^{\circ}$; (b) H4, $\phi=0$ and $\phi=45^{\circ}$; (c) Veloce. with $\phi=2 \pi$, screw lead $h=20$ and $h=50$; (d) V4, $\phi=0$ and $\phi=45^{\circ}$. 


\section{Conclusions}

This paper presents the transmission analysis for a class of four-limb parallel Schönflies-motion robots with articulated mobile platforms, closely in connection with two pressure angles derived from the forward and inverse Jacobian matrices, wherein the determinant of the forward Jacobian matrices was simplified in an elegant manner, i.e., the scalar product between two vectors, through the Laplace expansion. The cosine function of the pressure angles based indices are defined to evaluate the transmission quality. It appears that the robot with the screw-pair-based mobile platform, namely, the Veloce., is the best in terms of transmission quality for any orientation of the mobile-platform.

Acknowledgements The reported work is partly supported by the Fundamental Research Funds for the Central Universities (DUT16RC(3)068) and by Innovation Fund Denmark (137-2014-5).

\section{References}

1. Adept Quattro Parallel Robots. http://www1.adept.com/main/ke/data/Archived/Quattro/sQuattro_UG.pdf

2. Penta Veloce. http://pentarobotics.com/products/\#brochure

3. Altuzarra, O., Hernandez, A., Salgado, O., Angeles, J.: Multiobjective optimum design of a symmetric parallel Schönflies-motion generator. ASME J. Mech. Des. 131(3), 031,002 (2009)

4. Amine, S., Masouleh, M.T., Caro, S., Wenger, P., Gosselin, C.: Singularity conditions of 3T1R parallel manipulators with identical limb structures. ASME J. Mech. Robot. 4(1), 011,011 (2012)

5. Angeles, J.: Is there a characteristic length of a rigid-body displacement? Mech. Mach. Theory 41(8), 884-896 (2006)

6. Company, O., Krut, S., Pierrot, F.: Internal singularity analysis of a class of lower mobility parallel manipulators with articulated traveling plate. IEEE Trans. Robot. 22(1), 1-11 (2006)

7. Kim, S.G., Ryu, J.: New dimensionally homogeneous Jacobian matrix formulation by three end-effector points for optimal design of parallel manipulators. IEEE Trans. Robot. Autom. 19(4), 731-736 (2003)

8. Merlet, J.P.: Jacobian, manipulability, condition number, and accuracy of parallel robots. ASME J. Mech. Des. 128(1), 199-206 (2006)

9. Pierrot, F., Company, O.: H4: a new family of 4-dof parallel robots. In: IEEE/ASME Inter. Conf. Advan. Intell. Mecha., pp. 508-513 (1999)

10. Pierrot, F., Nabat, V., Company, O., Krut, S., Poignet, P.: Optimal design of a 4-dof parallel manipulator: From academia to industry. IEEE Trans. Robot. 25(2), 213-224 (2009)

11. Wang, J., Wu, C., Liu, X.: Performance evaluation of parallel manipulators: Motion/force transmissibility and its index. Mech. Mach. Theory 45(10), 1462-1476 (2010)

12. $\mathrm{Wu}, \mathrm{G}$. : Kinematic analysis and optimal design of a wall-mounted four-limb parallel Schönflies-motion robot for pick-and-place operations. J. Intell. Robot. Syst. (2016)

13. Wu, G., Bai, S., Hjørnet, P.: Architecture optimization of a parallel schönflies-motion robot for pick-and-place applications in a predefined workspace. Mech. Mach. Theory 106, 148-165 (2016)

14. Xie, F., Liu, X.: Design and development of a high-speed and high-rotation robot with four identical arms and a single platform. ASME J. Mech. Robot. 7(4), 041,015 (2015) 